\title{
Ueber das Vorkommen von Entzundung der Iris und Cornea bei Diabetes mellitus.
}

Von

Dr. F. Wiesinger in Gottingen.

Werfen wir einen Blick auf das Vorkommen von Augenerkrankungen bei Diabetes mellitus, so sehen wir Augenleiden der verschiedensten Art als hăufige Symptome dieser Krankheit auftreten. Vor allem zu erwähnen ist die Cataract, die als pathologisehes Vorkommniss bei Diabetes mellitus bereits seit etwa vier Decennien in die Lehrbücher übergegangen und damit allgemein anerkannt ist.

Ferner kennen wir eine Reihe von Beobachtungen über Netzhaut- und Glaskörperblutungen, über Retinitis apoplectica mit. und ohne Degenerationsheerde der Netzhaut. Der Zusammenhang dieser Augenleiden mit Diabetes mellitus geht klar daraus hervor, dass bei allen Angaben erwähnt wird, dass Jahre lang vor den betreffenden Netzhautaffectionen die charakteristischen diabetischen Erscheinungen aufgetreten seien. Besonders bei weit gediehenen, schweren Fällen von Diabetes mellitus kommen diese Netz- 
hauterkrankungen vor, bei welchen nach Prof. Leber*) zum Theil der Diabetes sellost als Ursache zu betrachten ist, zum Theil eine durch ihn hervorgerufene Nephritis oder auch beide gleichzeitig.

Als weitere Augenleiden bei Diabetes mellitus sind Amblyopien und Sehnervenerkrankungen zu erwahnen. Für den Zusammenhang mit Diabetes spricht wohl an besten die Wirksamkeit der anti-diabetischen Behandlung. Prof. Leber**) sagt darüber Folgendes: „Wenn es gelang, das Grundleiden zu bessern oder zu heilen, so wurden auch die überraschendsten Ruckbildungen und Heilungen der Amblyopie erreicht und zwar innerhalb kurzer Zeit. Eine vollständige Heilung ist nicht nur bei geringgradiger Sehstorung möglich, sondern selbst in so weit gediehenen Fällen, dass bei anderer Ursache kaum mehr an eine bedeutende Besserung gedacht werden könnte."

Was die Sehnervenleiden bei Diabetes anbetrifft, so scheint die bei weitem grössere Mehrzabl der Fälle ohne erhebliche cerebrale Stơrungen zu verlanfen und entfällt auf das directe Abhängigkeitsverhältniss von einem idiopathischen, nicht cerebral bedingten Diabetes.

Ferner gehören noch zu den Augenerkrankungen beiDiabetes mellitus Augenmuskellähmungen, die in Accommodationslähmung ond Mydriasis oder in Lahmungen der äusseren. Augenmuskeln mit Doppeltsẹen besteben können.

Erst in jüngster Zeit gemachte Beobachtungen über das Vorkommen von Keratitis and Iritis vermehrea noch die Anzahl derAugenleiden, die wir bei Diabetes auftreten sehen. Diese Thatsache kann uns nicht überraschen. Haben wir doch oben schon eine Reihe von Augenerkrankungen bei Diabetes mellitus kennen gelernt, weshalb sollten nicht auch

*) v. Graefe's Archiv für Ophthəlmologie, Bd. XXI, Abth. 3, p. 58.

**) L. c. p. 91 . 
Entzitudung der Iris und Cornea bei Diabetes mellitus. 205

zuweilen die überaus empfindlichen Gebilde wie Cornea und Iris ergriffen werden?

Fälle von Keratitis bei Diabetes mellitus sind an hiesiger Augenklinik bisher noch nicht beobachtet, dagegen kam in einer ganz ansehnlichen Zahl von Fällen Iritis vor, theils for sich allein, theils mit anderen diabetischen Erkrankungen der Augen combinirt. Als besonders erwähnenswerth ist dabei das Auftreten von Hypopyon anzufübren, zumal da die primäre, nicht von Keratitis fortgeleitete eitrige Iritis nicht $\mathrm{zu}$ den häufigen Vorkommnissen. gehört. Wiederholt wurde in solehen Fallen der Diabetes erst dadurch diagnosticirt, dass die ungewöhnliche Form des Augenleidens nach früheren Erfahrungen die Untersuchung des Urins veranlasste, auch ohne dass die charakteristischen Symptome des Diabetes vorhanden waren, wie dies auch bei den übrigen diabetiscben Erkránkungen des Auges bekanntlich hänfig genug vorkommt. Durch die Güte des Herrn Prof. Leber wurde mir die Veröfientlichung der betreffenden Krankengeschichten freundlichst überlassen.

Doch bevor wir uns den hiesigen Beobachtungen selbst zuwenden, wollen wir der Vollständigkeit und Uebersicht wegen die vereinzelten Mittheilungen in der Literatur über Keratitis- und Iritisformen bei Diabetes mellitus näher betrachten.

Was zunächst die Keratitis bei Diabetes mellitus anbetrifft*), so liegen darüber folgende Beobachtungen vor.

I. Die erste mir bekannte Mittheilung darüber findet sich in der Gazette médicale von 1857 von Leudet. Es handelt sich darnach um eine Frau von 32 Jahren, die unter den charakteristischen Symptomen von Diabetes an linksseitiger Keratitis erkrankte. Zugleich bestand eine Läbmung des

*) Eine Notiz von Coundouris (Thèse de Paris 1883), wonach $1830 \mathrm{Himly}$ zuerst Keratitis bei Diabetes mellitus beschrieben babe, hat sich nicht bestätigt gefunden. 
dritten und fünften Hirnnerven der linken Seite. Bei der Autopsie stellte sich heraus, dass es sich um eine syphilit. Affection der Meningen an der Hirnbasis handelte, woraus sich in ungezwungener Weise die Lähmung des Trigeminus erklärt. Dass aber bei Verletzung resp. Lähmung des Trigeminus und damit zusammenhängender vollständiger Anästhesie der Hornhaut, wodurch das Auge bei Einftuss äusserer Schädlichkeiten sich nicht mehr durch den Lidschluss schutzt, Koratitis auftritt, ist längst anerkannt. Es bleibt somit ungewiss, ob die Keratitis die Folge des Diabetes oder vielmehr der Trigeminuslähmang gewesen war; wahrscheinlich dürfte sie als neuroparalytische aufzufassen sein. Jedenfalls kann daher dieser Fall nicht herangezogen werden, um das Vorkommen von Keratitis in Folge von Diabetes zu beweisen.

II. Nach Prof. Leber*), soll r. Graefe bereits in den 60er Jahren auf das Vorkommen von eitriger Keratitis bei Diabetes in seinen klinischen Vorträgen aufmerksam gemacht haben.

III. Später führt Pana s* $^{*}$ Albuminurie and Diabetes mellitus als praedisponirende Ursachen der eitrigen Keratitis an und berichtet, dass er noch vor kurzem den sehadlichen Einfluss des Diabetes in der genannten Richtung selbst bei wohlhabenden und anscheinend gesunden Individuen $\mathrm{za}$ constatiren Gelegenheit gehabt habe.

Ferner hat Galezowsizi in Paris verschiedene Fälle von Keratitis bei Diabetes beschrieben.

IV. Der erste Fall wurde von ihm im Jahre 1879 publicirt im „Recueil d"ophth."****) Er betrifft einen Mann von 58 Jahren, mit schon lange bestehender beiderseitiger Myopie von 5D. Seit zwei Jahren gleichseitige Hemianopsie nach rechts, plötzlich entstanden. Sait 8 Tagen Entzündung am linken Auge mit beträchtlicher Sehstörnng, Finger werden nur auf etwa 1 Meter gezählt. Die Cornea ist matt, besonders in der unteren Hälfte, mit mehreren kleinen, obertächlichen Substanzvérlusten. Lebhafte pericomeale Injection und starke Lichtscheu. Keine Synechien, träge Pupillarreaction. Absolute Anästhesie der Cornea. Das Gesichtsfeld fehlt rechts nach aussen, links nach

*) v. Graefe's Archiv f. Ophth., Band XXI, Abth. 3, p. 22.

*) Panas, Leçons sur les Kératites. Paris 1876, p. 158.

* Der Fall ist reprodncirt in der Thêse ron Ooundouris, aus der wir die Angaben in Ermangelung des Originals entuehmen. 
Entziündung der Iris und Cornea bei Diabetes mellitus. 207

innen vollständig. Der Urin enthält beträchtliche Mengen von Zacker. Die Diagnose wurde auf Keratitis glycosurica gestellt. Durch antidiabotische Diät, Atropin nnd Eserin abwechselnd, Schröpfköpfe und warme Umschläge trat eine stetige Besserung des Zustandes ein, so dass Pat. nach Verlauf von einigen Wochen entlassen werden konnte. Die Hornhaut hatte ihre Durchsichtigkeit und Empfindlichkeit grösstentheils wieder erlangt.

Leider fehlt die Angabe, $a b$ die Sensibilitätsstörung auf das Auge beschränkt war oder eine Theilerscheinung einer Trigeminuslähmung darstelite, woran bei der Complication mit gleichseitiger Hemianopsie wohl gedacht werden kann, da letztere die Anwesenheit eines intracraniellen Leidens mit Sicherheit beweist. Es bleibt also fraglich, ob die Keratitis als direct ron dem Diabetes abhängig gedacht werden darf, oder ob, ähnlich wie in dem Leudet'schen Falle, die Keratitis sowohl als die Hemiopie auf dieselbe centrale Störung zurückzuführen ist.

V. Eine zweite, nur ganz summarisch mitgetheilte Beobachtung Galezowski's*) von Keratitis bei Diabetes stammt aus dem Jahre 1880. Er constatirte bei einer diabetischen Frau ron 64 Jahren linkerseits ein progredientes Hornhautgeschwür, das sich zugleich mit einem fieberhaften Zustand entwickelt hatte und mit Hinterlassung von Hornhauttrübung zur Heilung kam.

VI. Ebenso kurz ist die Mittheilung über den dritten Fall Galezowski's.**) Hierbei handelt es sich um einen 63jahrigen Diabetiker, der im Verlauf von 8 Monaten zweimal von schwerer eitriger Keratitis mit Hypopyon befallen wurde, welche den Hornhautschnitt nöthig machte.

VII. Folgenden Fall von Keratitis bei Diabetes beobachtete Bellouard***) an der. Klinik von Panas zu Paris. Am 28. Juni 1882 verlor ein Kutseher von 33 Jahren plötzlich nach heftigen Kopfschmerzen das Bewusstsein. Zugleich bemerkte man beiderseitige Injection der Augen, besonders des linken. Nach vergeblicher Behandlung einer schweren Keratitis im Hôpital Necker wurde Pat. in Panas Klinik aufgenommen, wo am 13. Juli 1882 folgender Zustand notirt wurde:

*) Conndonris, Thêse de Paris 1883, p. 48.

**) Journal de thérapeutique, mars 1883.

***;) Coundouris, loc. cit, p. 46. 
Heftige Ciliarneurose. Augendruek sehr gesteigert. Die Cornea wird won einem grossen, unregelmässigen, an den Rändern gezackten Geschwür eingenommen. Die vordere Kammer voll Eiter. Spaltong des Geschwürgrundes nach Saemisch, worauf sofortige Erleichterung eintritt. Urin stark zuckerhaltig. Ueber den Ausgang der Krankheit wird leine Wittheilung gemacht. Auch hier ist in der Krankengeschichte die Möglichleit, dass es sich - in Anbetracht der durch den apoplectiformen Anfall documentirten cerebralen Störung um eine neuroparalytische Keratitis gehandelt habe, nicht ausdrücklich ausgesehlossen.

VIII. Eine kurze Notiz findet sich noch nach Coundo uris*) bei Desprès (Chirurgie journalière) über oine diabotische Frau mit eitriger Infiltration und Geschwürsbildung beider Corneae. Das linke Auge ging durch Perforation mit Irisvorfall und späterer Staphylombildnng verloren. Das andere besserte sich.

IX. Der letzte Fall von Keratitis bei Diabetes wurde von Coundouris**) selbst an der Panas'schen Klinik beobachtet.

E. Trouillier, 53 Jahr alt, leidet seit 4 Jahren an ausgesprochenen diabetischen Beschwerden. Die tägliche Urinmenge betrug ror 2 Jahren 6 Liter bei 11,5 pCt. Znckergehalt. Nach einer Knr in Vichy verminderten sich die diabetischen Symptome und fiel die Lrinmenge ant 2,5 Liter bei $3,5 \mathrm{pCt}$. Zuckergehalt, um spater wieder etwas zu steigen. Eiweiss war nicht vorhanden. Seit einem halben Jahr nahm das Sehvermögen am linken Auge ab and trat an demselben etwas Lichtsoheu bei Arbeiten mit künstlichern Lichte auf.

Vor sechs Wochen trat Entzündung an diesem Auge auf nit stärkerer Lichtschea, aber ohne erhebliche Schmerzen. Dieser Zustand besserte sich nach 5 Tagen, aber am folgenden Tage war das Sehrermögen stärker getrubt. Seit 8 Tagen Nachts Ciliarneurose.

Stat. praes, am 10. Juli 1883. Keine Abmagerung. Krafte gut, bei erböhtem Hunger und Durst. Conjunctiva des linken Anges injicirt und etwas chemotiseh. Bei sehräg auffallendem Licht sieht man die ganze Hornhaut getrübt, am stärksten im Centrum. Bei directer Beleuchtang bemerkt man in der oberen Partie der Cornea eine dunkle Punktirung. Die

*) loc. cit., p. 48 .

**) loc. cit., p. 60 . 
Entzündung der Iris und Cornea bei Diabetes mellitus. 209

Pupille ist etwas unregelmässig, die untere Partie der Iris etwas verfärbt. Die Empfindlichkeit der Hornhaut hat nicht abgenommen. Der Urin enthält こ̌,6 pCt. Zucker bei einer 94stündigen Menge von 3 Liter.

Nach Eịnträufeln von Atropin, warmen Emschlägen und innerlichem Gebrauch von KJ verschwinden die Hauptsymptome nach wenigen Tagen, nur die centrale Trübung der Cornea bleibt noch bestehen.

Aus den folgenden Bẹmerkungen geht hervor, dass die Krankheit als parenchymatöse Keratitis nicht syphilitischen Ursprungs aufgefasst wurde, die aber in Anbetracht der periorbitären Schmerzen und der Unregelmässigkeit der Pupille jedenfalls mit Iritis complicirt war. Dieser Umstand, sowie die auffallend rasche Besserung ohne weitere Behandlung des Diabetes nur durch Gebrauch von KJ macht es fraglich, ob wir es hier wirklich mit einer durch Diabetes erzeugten Keratitis zn thon gehabt haben, oder ob dem Leiden Syphiliszu Grunde gelegen hat.

Dieser letzte Fall von Keratitis bei Diabetes mellitus, der mit Iritis complicirt war, bildet einen passenden Uebergang zu den Iritisformen bei Diabetes, denen wir uns nun zuwenden wollen.

Auch hierbei sollen zunächst die wenigen Mittheilungen der med. Literatur über derartige Krankheitsfälle Berücksichtigung finden.

I. Die erste Beobachtung der Art stammt aus dem Jahre 1863 von Demarquay und ist mitgetheilt von Marchal de Calvi*). Es handelt sich um eine corpulente, über 50 Jahre alte Frau, die an einer hartnäckigen Iridochorioiditis litt. Nach Application eines Vesicans im Nacken war eine ausgedehnte und tiefe brandige Phlegmone entstanden, wegen deren die Kranke auf Demarquay's Abtheilung verlegt wurde. Hier wurde Diabetes diagnosticirt. Die Behandlung war aber vergeblich, die Patientin starb nach wenigen Tagen an Entkräfttung.

II. Einen zweiten Fall von Iritis bei Diabetes mellitus beobachtete Noyes**) im Jahre 1869.

*) Marchal de Calvi, Noivelle théorie du diabète p. 414; citirt bei Coundouris p. 55 .

*) H. D. Noyes, Transact. of the Amer. Ophth. Soc. 4. \& 5. ann. meet: New-York 1869, p. 71-75.

v. Graefe's Archiv für Ophthalmologie, XXXI. 4. 
60 jänrige Frau. Vor drei Jahren Diabetes diagnosticirt; Besserung durch Fleisehdiăt und Opium. Im letzten Sommer doppelseitige Iritis, die mit Synechien heilte. Sehrermögen, rorher schon etwas vermindert, war der optischen Storang entsprechend. Beiderseits Retinitis mit weissen Degenerationsherden, die im weiteren Verlauf ganz das Aussehen der Retinitis albuminurica annahm. Der Urin enthielt bei längere Zeit fortgesetzter Beobachtung immer beträchtliche Mengen Zucker, aber niemals Eiweiss.

III. Einen weiteren Fall theilt Wickersheimer*) mit ans der Abtheilung von Chanvel in Val-de-Grâce unter der Bezeichnung Iridochorioiditis glycosuriea.

Dezumeaux, Soldat im 1. Zuavenregiment, 37 Jahre alt, bemerkt seit etwa einem Jahr starken Hanger und Durst, sowie eine Abnahme seiner Kräfte, die sich so steigerte, dass or Aufnabme im Hospital suchen masste und nachler nach Frankreich zurückliehrte. Der Gelalt an Zucker soll 20,3 pCt. betragen baben bei einer Urimmenge von 4 Ltr. pro 24 St. Kein Eiweiss. Anfang December 1873 trat Entzündung des 1. Auges mit Lichtscheu, Chemosis und heftiger Ciliarneurose auf. Daranf nalm in wenigen Tagen die Sehkraft so ab, dass Patient nur noch die Contouren von Menschen und grösseren Gegenständen sah. Das rechte Auge war vollständig gesund. Nach einiger Zeit hatten Injection und Schmerzen etwas abgenommen und die Pupille reagirte etwas besser auf Licht. Starke Verfärbung der Iris. Hornhaut sowohl wie Linse sind durchsichtig. Ophth. Befund: Angenhintergrund graulich getrübt. Papille und Gefüsse sind rerschleiert, Venen von ungleicher Weite, Arterien nicht zu sehen. Flottirende Trübung in Glaskörper. Gesichtsfeld schläfenwärts etwas beschränkt. Sehschärfe auf $1 / 40$ herabgesetzt.

Veber den weiteren Verlauf der Erkrankung ist nichts bekannt.

IV. 1879 batte Galezowki**) Gelegenheit, einen Fall von Iritis bei Diabetes zu beobachten. Ein Mann von 60 Jahren stellte sich am 14. Februar 1879 in der Klinik vor mit linksseitiger Iritis und mit hintern Synechien in beiden Augen. Schmerzanfăllo in der Nacht. Vor zwei Jahren batte er am

*) Wickersheimer, Thèse de Paris. 1874.

**) Ooundouris, Thèse de Paris 1883, p. 53. 
rechten Auge Iritis gehabt, die innerhalb 8 Tagen geheilt war. Im Alter von 25 Jahren hatte er einen Anfall von acutem Gelenkrbeumatismus. Seit 1866 ist er Diabetiker. Die Zuckermenge betrug damals nach wiederholten Analysen $9 \mathrm{pCt}$. bei einer 24 stïndigen Drimmenge von 4 Ltr. Fr beobachtet seit der Zeit antidiabet. Diät und seit 2 Jahren waren nur noch Spuren von Zucker nachzuweisen. Bereits nach 14 Tagen ist die Iritis bedentend besser und nach Verlauf von 3 Monaten völlig geheilt, obwohl der Urin wieder Zucker enthält.

V. Am 12. März 1879 kam eine Patientin von 28 Jahren wegen Sehstörung in die Klinik von Dr. Abadie*). Es handelte sich um eine beginnende Cataract bei Diabetes mellitus, wegen dessen die Kranke schon lange in Behandlung war. Im weiteren Verlauf verschlimmerte sich trotz der strengsten Diät der Allgemeinzustand, und der Urin enthielt enorme Mengen von Zucker. Patientin magerte stark ab und füblte sich kraftlos, so dass nicht an Extraction der inzwischen matur gewordenen Cataract gedacht werden konnte. Mittlerweile trat am r. Auge heftige Entzündung mit lebhaften Schmerzen auf. Die Iris war verfärbt und es hatten sich im ganzen Umfang der Pupille Synechien gebildet. Der intraoculäre Druck war gesteigert. Es handelte sich offenbar um eine Iridochorioiditis mit Glancom. Nach einigen Tagen erkrankte auch das 1. Auge, aber weniger heftig. Der Allgemeinzustand verschlimmerte sich noch weiter, so dass Patientin wobl bald ihrem Leiden erlegen ist.

VI. 1881 wurde ron Umman**) ein weiterer Fall von Iritis bei Diabetes beobachtet bei einem Priester von 69 Jahren, aber kräftiger Constitution, der schon seit 10 Jahren an Diabetes litt, mit allen charakteristischen Symptomen und hohem Zuckergehalt. Bei der Vorstellung litt der Pat. schon seit einigen Tagen an heftiger Iritis, ansserdem an Blasenkatarh mit Fieber.

12. September. St. pr.: Sehschärfe für das Alter normal, weder eine Spur von Retinitis noch von Cataract. Patient klagt seit 11 Uhr morgens sehr über sein reehtes Auge. Starke Lichtscheu and circumcorneale Injection, heftige Schmerzen. Die Iris ist matt, die Pupille stark verengt und reactionslos; grosses Hypopyon. Am nächsten Tage haben

*) Coundouris, . 52 .

**) France médicale 1881, citirt bei Coundouris, p. 55 . 
sich die verschiedenen symptome noch gesteigert, spater bljeb der Zustand derselbe. Der Patient ging nach 4 Tagen in Folge der heftigen Cystitis unter urämisehen Erscheinngen zu Grunde, ohne dass sich die Iritis gebessert hatte.

VII. 1883 beschreibt Galezowski *) einen Fall ron Iritis bei Diabetes neben Retinitis haemorrhagica. Patient ron 35 Jahren litt seit einiger Zeit an Sehstörung durch Retinitis haemorrhagica. Zugleich wurde ein Zuckergehalt seines Urins von 8 pCt. constatirt. Ein Jahr später trat an einem Auge plötzlich Tritis auf mit unerträglichen Schmerzen, die durch Paracentesen gemildert wurden. Nach einem Monat entwickelte sich unter neven, heftigen Schmerzen an demselben Auge ein Glaucom, weswegen die Iridectomie ansgefürt wurde. Die nach 3 Wochen ron neuem auftretenden sehr heftigen Schmerzen führten endlich zur Enucleatio bulbi, wodurch die Schmerzen dauemd beseitigt wurden und dem andern Ange seine rolle Sehkraft erhalten blieb.

VIII. Ein letzter Fall endlich von Iritis wird uns von Coundouris *⿻) mitgetheilt.

Am 4. April 1883 stellte sich ein 54jähriger Mann in der Klinik ron Galezowski vor. Sein Vater war vom 40. Jahre an diabetisçh nnd starb mit 75 Jahren; sein gleichfalls diabetischer Oheim wurde 90 Jahre alt; anch sein Broder leidet an Diabetes. Schon vor 12 Jahren bemerkte er vermehrten Durst und Urin. Die Analyse des Urins ergab eine grosse Menge won Zueker. For 2 Jahren trat linkerseits zuerst Iritis auf ohne Schmerzen und Lichtscheu, die nach einem Monat ausheilte. Schon vor dieser Iritis begann seine Sehschärfe abzunehmen und oft sah er Kreise um eine Flamme herum. Am 1. April trat plötzlich auch am $r$. Auge Iritis auf mit lebhaften Schmerzen und Lichtschen. Das $r$. Auge zeigt ausgesprochene pericorneale Injection, die Pupille ist unregelmüssig durch hintere Synechien. Keine Abmagerung. Oefters unbestimate Schmorzen in den Gliedern, häufiges Kriebeln, zuweilen anch Krämpfe, Appetit nicht gesteigert. Urin enthält 6,2 pCt. Zucker bei einer 24 st. Nenge ron 2 Liter. Behandlung mit Schröpflöpfen and Atropin bleibt erfolglos, dagegen rasche

*) Journal de Thérap. 1883; wieder mitgetheilt pon Coundouris p. 51.

*) loc. eit. p. 54 . 
Entzündung der Iris zud Cornea bei Diabetes mellitus. 213

Besserung darch Einträuflung von Pilocarpinlösung. Nach Verlauf ron 3 Wochen war die Iritis geheilt.

Ich wende mich jetzt zur Mittheilung der

\section{Beobachtungen aus der Göttinger Augenklinik.}

Fall I.

Iritis adhaesiva, Amblyopia ex atroph. n. opt. part., Diabetes. mellitus.

Konrad L. aus Lichtenau, 57 Jahre alt, lat im Jahre 1853 4 Monate lang an sehr ausgesprochener Intermittens tertiana gelitten; Chinin half nur vorubergehend, scheint aber nicht consequent genommen worden zu sein; seitdem traten keine Anfälle mehr auf. Im August 1867 wurde er als Gutsverwalter in der Provinz Posen von einem Augenleiden ergriffen, das ihn bei zunehmender Sehstörung veranlasste, Dr. Cohn in P'osen zu consultiren. Dieser constatirte schon damals Amblyopie und Diabetes mellitus. Darauf wandte sich Patient an v. Gra efe in Berlin, auf dessen Rath er zur Kur nach Karlsbad ging, wo er vom 6. November bis 25. December verblieb, ohne aber eine Besserung seiner Amblyopie zu erzielen. Diabetische Erscheinungen sollen weder damals, noch später vorhanden gewesen sein. In Karlsbad sei von einem Apotheker 7 pCt. Zucker im Harn gefunden worden, nach nur zweitägiger Kur habe aber die Untersuchung in einer anderen Apotheke ein ganz normales Verhalten des Harns, auch normales spec. Gewicht ergeben. In die v. Graefe'sche Klinik zurückgekehrt, wurde Patient bald nach seiner Aufnahme als ein Fall von Amblyopie, wahrscheinlich in Folge von Diabetes mellitus vorgestellt, worüber er recht genaue Angaben macht. Andere Ursachen, insbesondere Alkoholintoxication, konnten ausgeschlossen werden; die Sehschärfe habe etwa $1 / 5$ betragen, das Gesichtsfeld soll frei gewesen und ausser etwas Hyperämie mit dem Augenspiegel nichts $A b n o r m e s$ gefunden sein. Der Zuekergehalt des Harns soll ca. 1,5 pCt. betragen haben. Trotz diabet. Diät und ableitender und diaphoretischer Behandlung wurde keine Besserung des Sehvermoggens herbeigeführt, sodass Patient nach zwei Monaten in demselben Zustand, wie er gekommen, die Klinik wieder verliess. Der Augenschwäche wegen musste er seine Stelle als Gutsverwalter aufgeben. - Erst im Winter 1870/71 trat ganz von selbst eine allmählig fortschreitende Bésserung ein, sodass Patient (mit Hülfe einer schwachen Convexbrille) 
wieder schriftliche Arbeiten machen und im Jahre 1873 is seinem neuen Wohnort Lichtenau die Stelle eines Cassirers übernehmen konnte, die er bis vor wenigen Wochen ungestört versah. Eine Behandiung des Augenleidens, resp. des Diabetes hatte nicht mehr stattgefunden, ebensowenig hatte Patient diabetische Diät weiter beobachtet. Nur will er nie viel süsse Speisen, keinen Zucker und wenig Kartoffeln genossen haben. Das Allgemeinbefinden ist in den letzten $10 \mathrm{Jahren}$ stets gut gewesen.

Jetzt vor 3 Wochen stellte sich linksseitige Augenentzündung mit Abnahme des Sehvermögens ein (letzteres avch reehts, doch in geringerem Grade), wobei zugleich von dem Arzt Zucker im Harn nachgewiesen wurde.

Patient erinnert sich nachträglich, dass damals sein Durst doch etwas vermehrt gewesen sei; or habe vor etwa 14 Tagen die tägliche Urinmenge zu 2y/4 Liter bestimmt. Er wurde ohne Erfolg mit Atropin, Zinklosung und Natr. salicyl. innerlich behandelt; letzteres Mittel warde nur mit Widerwillen genommen und schlecht vertragen.

5. December 1879. Stat. praes.: R. A. Aeusseres normal, abgesehen yon einem schmalen Pigmentsaum am Pupillarrand der Iris obne Synechien. Ophth. Papille weisslich rerfärbt, besonders die temporale Halfte. Gefässe normal. L. Lider etwas ödematös, tiefe, mässig starke Ciliarinjection. Kammerwasser, besonders in der unteren Hälfte leicht getrübt. Pupille von gleicher Weite wie R, nicht adhaerent, zeigt denselben etwas unregelmässigen Pigmentsaum wie dort. Bild des Augengrundes ziemlich stark versehleiert, doch lässt sich ebenfalls eine Verfärbung der Papille feststellen. Augendruck beiderseits etwas hoch.

Patient lrlagt über Druckgefühl im linken Ange, besonders beim Bücken; zeitwoise Thränen ete.

R. $\mathrm{S}^{20} / 200$. Mit +3 D N.12 (J) mühsam. Se frei.

L. Finger in $12^{i}$ gezählt, mit $+3 \mathrm{D}$ Worte von $\mathrm{N} .16(\mathrm{~J})$ Se frei. Farben werden richtig angegeben.

Urin enthallt viel Zucker.

Der Kranke wurde zunächst zur genaueren Bestimmung des Zuckergehaltes auf gemischte Kost gesetzt und die Iritis in Anbetracht des etwas hohen Augendruekes mit Eserineinträufelungen (2 mal täglich) behandelt.

6. December. Stat. idem., nur 1. Andeutung leichter 
Entzündnng der Iris und Cornea bei Diabetes mellitus. 215

Trübungsstreifen der Cornea. Pupille gleich weit wie R., nach Eserm enger. Druck heute beiderseits nicht erhöbt.

24stündige Urinmenge $2746 \mathrm{Ccm}$, Titrirung mit $\mathrm{F}$ e hling'scher Lösung ergiebt 4 pCt. Zucker (pr. dio 109,8 Gramm).

7. December. L. Trübung wohl etwas weniger, R. Sehr leichte Injection der Conjunctiva sclerae. Strenge Fleischdiät.

8. December. Diät und Eserin wie bisher. L. Inject. etwas geringer; Trübung der Cornea und des Kammerwassers ebenfalls entschieden weniger geworden. Augendruck normal. Sehstörung auch etwas gebessert. Urin enthält noch $4 \mathrm{pCt}$. Zucker, aber 24 st. Menge nur $1640 \mathrm{Ccm}$, daher. nur 65,6 Gr. pro die. Kein Eiweiss.

9. December. Augen ziemlich unverändert. Zuckergehalt 1,8 pCt. 24 st. Menge $1500 \mathrm{Ccm}, 27,0 \mathrm{Gr}$. Zucker pro die.

10. Décember. Kein Eiweiss. Zuckergehalt 1,3 pCt, 24 st. Menge $1644 \mathrm{Ccm}$, also $21,37 \mathrm{Gr}$. pro die. Hornhaut und Kammerwasser entschieden klarer.

In den nächsten Tagen sinkt bei Fortsetzung der Fleischdiät der Zuckergehalt noch stetig weiter.

Am 15. December wurde noch Karlsbader Wasser eine Flasche täglich verordnet.

Am 16. December 'Zuckergehalt 0,93 pCt. bei $11,7 \mathrm{Gr}$. täglicher Menge, Spuren von Eiweiss.

Am 17. December ist das Auge noch immer injicirt. Lider etwas mehr ödematös. Nachts Schmerz. Trübung geringer; nach innen von der Hornhautmitte ein schon längere Zeit bemerktes weisses Pünktehen, das der hinteren Wand aufgelagert scheint.

Eserin vor einigen Tagen wegen Reizerscheinungen ausgesetzt, statt dessen warme Umschläge und 3 Mal täglich 0,3 Chinin.

18. December. Subjective Besserung. Schmerzen verschwunden. Obj. stat. idem.

19. December. Wiederauftreten von Schmerzen. Kleine Blutung in die vordere Augenkammer. Pupille frei davon. Im Urin nur Spuren von Zueker; kein Eiweiss, 24 st. Menge $2560 \mathrm{Gr}$. Ord. Heurtelonp. 1/2 Cyl.

20. December. Keine Schmerzen, Blutung nahezu resorbirt. 2 std. Atropin. I. Pupille auf Atropin nur wenig erweitert, nnregelmassig. Spuren von Zucker.

22. December. L. Injection und Kammerwassertrübung ent- 
schieden geringer. Chinin und Karlsbader Wasser fortgesetzt. Pupille trotz 2 stdl. Atropin nur mittelweit, riele feine Synechien. Im Urin weder Zucker noch Eiweiss.

24. December. L. Injection viel geringer, Trübung hat abgenommen. Pupille etwas weiter, aber noch stark und vielfach adhaerent. Keine Spur von Zucker.

26. December. Seit gestern 1 Weissbrödchen täglich. Urin absolut frei von Zncker. Injection geringer. Pupillarrand fingt an, sich mehr abzulösen. In der Mitte der Pupille zarte bräunliche Auflagerung.

27. December. L. Injection noch geringer. Pupille besonders in horizent. Richtung erweitert, doch noch vielfach adhaerent.

30. December. L. Injection fast ganz zurückgegangen. Iris noch etwas grünlich im. Vergleich zu R. Cornea und Kammerwasser klar, nur auf ersterer noch das feine Pünktchen. Pupille durch Atrop. noch immer unvollkommen erweitert; feine bräunliche Pigmentauflagerungen und hintere Synechien.

Ophth. Bild fast ganz klar; leichte aber deutliche Sehnervenverfärbung, die R. mellr ausgesprochen ist.

Patient über die Besserung sebr exfreut, giebt an, wieder nahezu. so gut zu sehen wie früher. R. \& $20 / 200$; mit $+3 \mathrm{D}$

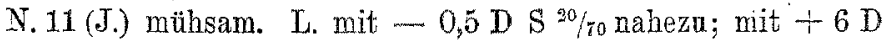
(Atropin) N. 3 J. Urin frei ron Zucker.

31. December 1879. Bei nochmaliger genanerer Prüfung des Farbensinns stellt sich jetzt am r. Auge ein kleines horizontal ovales paracentrales Farbenscotom heraus; im übrigen Gesichtsfeld werden die Farben ziemlich richtig wnterschieden, doch in sehr kleinen Stückchen auch etwas matt. Am l. Auge ist dasselbe Verhalten, aber noch weniger ausgesprochen. Beim Vorlegen grösserer Farbenproben werden beiderseits alle Farben richtig angegeben.

Entlassen mit der Verordnung, noch 3 Wochen Karlsbader Wasser zu gebranchen, und Atropin einzuträufeln.

Patient stellte sich auf Wunseh am 16. Janiuar 1880 wieder vor. L. nach aussen mehrere bintere Synechien; aut der Kapsel bräunliches Pigment abgelagert. Auge noch leicht injicirt. Urin ohne Zucker. Ophth. Verfürbung wie früher. Nach Brief rom 5. März 1880 hat sich das Sehvermögen noch gebessert und soll besser sein als vor der Iritis. Patient hat Fleisehdiät fortgesetzt und täglich nur $1 \frac{1 / 2}{2}$ Semmeln gegessen. Sein Körpergewicht hat seit 1. Januar um $6^{1 / 4}$ Pfd. 
Entzïndung der Iris und Cornea bei Diabetes mellitus. 217

zugenommen. Der übersandte Urin enthält aber eine nicht unbeträchtliche Menge Zucker.

Patient berichtet gelegentlich am 10. April 1883, dass es ihm seither vollkommen gut gehe bei fortgesetzter Diät. Das Körpergewicht habe auch in den letzten Jahren noch stetig zugenommen. Der übersandte Urin von 1,020 spec. Gewicht, zeigt starke Zuckerreaction, ist aber frei von Eiweiss.

Auf eine briefliche Anfrage berichtet der Kranke am 5. October 1885 ,über sein jetziges Befinden Folgendes: Sein Sehvermogen ist seit der Entlassung gut geblieben, er kann mit einer Convexbrille (1,5 D) seinen Berufsgeschäften nachgehen und hat keine Rückfälle von Entzündung gehabt. Das Allgemeinbefinden ist trotz der Zuckerkrankbeit, die seit 18 Jahren besteht, vollkommen gut, Patient ist ein rüstiger Fussgänger und hat mit 62 Jahren noch den Meissner bestiegen. Die Diät hat er ziemlich streng gehalten. Der übersandte Urin ron 1,030 spee. Gewicht enthalt 5 pCt. Zncker, aber kein Eiweiss.

\section{Fall II.}

Iritis fibrinosa, Diabetes mellitus, Albuminuria levis.

Hermann S. aus Blickershausen, $37 \mathrm{Jahr}$, kommt zur Behandlung am 3. Juli 1882. Kleines verwachsenes Individuum in Folge hochgradiger Rhachitis; untere Extremitäten verkümmert. Geht auf Krüeken. Keine sonstigen Klagen, nichts von Gonorrhoe oder von Lues nachzuweisen.

Beiderseits Augenentzündung, R. seit 8 Tagen, L. seit 3-4 Tagen. Beide Pupillen von einer membranösen Exsudation eingenommen, welche $\mathrm{L}$. die Pupille vollständig ausfüllt, R. sich an einem Rande etwas abzulösen beginnt. Starke Injection, mässige Verfärbung der Iris. Ord. Natr. salieyl. 2 mal täglich 2,0; Atropin.

5. Juli. Auffallend rasche Besserung und prompte Resorption der Pupillarmembranen, welche heute schon ganz verschwunden sind. Pupillen durch Atropin noch nicht vollständig und leicht unregelmässig erweitert, aber olne vorspringende Synechien.

7. Juli. Sehvermögen: R. mit $+1,5$ D s $20 / 100-70$, L. mit $+1,5 \mathrm{D} \mathrm{S} 2 \% 200$.

22. Juli. Bei weiterer Rückbildung der Veränderungen hat sich das Sehrermögen noch stetig gebessert. R. mit $+0,5 \mathrm{D}$ 
S $20 / 50$ nahezu, L. mit $+0,5 \mathrm{DS}{ }^{20} / 100-70$, mit $+1 \mathrm{D}$ jeders. N.3 (J.) ziemlich geläufig gelesen.

23. Juli. Entzündung ziemlich abgelaufen. Pupille war in der letzten Zeit nicht vollständig regelmässig, obwohl sie früher durch Atropin leidlich gut erweitert war. Deshalb eine Zeit lang Eserin eingeträufelt, das vor 2-3 Tagen wieder ausgesetzt wurde. Jetzt treten R. anf Atropin am medialenPupillarrand einige Synechien hervor. Pupillargebiet völlig frei nnd Angenhintergrund scharf zu sehen. Keine Glaskörpertrübungen, dagegen am r. Ange ein kleiner weisser Herd in der Retina, im umgekehrten Bild nach innen und etwas nach unten ron der Papille, ungefähr $1 \mathrm{P}$ entfernt, welcher ein Netzhautgefäss verschleiert.

Erst heute, wo Patient von seiner Iritis geheilt entlassen werden soll, wird gemeldet, dass derselbe während seines hiesigen Aufenthaltes einen ausserordentlichen Appetit zeigte und Nachts mehrmals aufstehen musste, um Crin zu lassen. Die Untersuchung des Urins ergiebt bei einem spec. Gewicht von 1,040 6 pCt. Zucker und daneben etwas Eiweiss. Pat. wurde mit entsprechenden Vorsehriften nach Hause entlassen, hat sich später nicht wieder vorgestellt und ist nach neulich eingezogener Erkundigung inzwischen rerstorben.

\section{Fall III.}

Iritis fibrinosa purulenta oc utr, Diabetes mellitus Phthisis pulmonum.

Eduard W., 47 Jahre, ans Lauterberg.

17. Juni 1881. R. Iritis acuta seit 8 Tagen. Kleines Hypopyon, starke Kammerwassertrubung, grünliche Verfärbung der Iris, membranöse Exsudation im Pupillargeliet, die ganze Pupille cinnehmend. Einige zarte Trübungsstreifen in der Cornea. Nach zweimal Atropin Pupille nicht weiter, ringsum an der Pupillarmembran adhärent. Keine erheblichen Schmerzen, mehr Gefühl von Fremdkörper im Ange. Patient will im vorigen Jahre heftige rheum. Schmerzen im 1 . Hüftgelenk und ofters leichtere Beschwerden in rerschiedenen Korpertheilen gehabt haben. Lues und Gonorrhöe in Abrede gestellt. Keine sonstigen Klagen. Die Affection wurde daher als Iritis rheumatica aufgefasst, Patient in die Klinik aufgenommen und mit Natr. salicyl., zweimal tägl, 2,0, behandelt. Atropin wurde nichț weiter eingeträufelt, um die alleinige Wirkung der Salicylsäure zu beobachten. 
19. Juni. Der Zustand hat sich gebessert. Hypopyon kleiner.

20. Juni. Auffallend rasehe Besserung. Hypopyon verschwunden. Kammerwasser im obern Theil ziemlich klar, nur nach unten noch leichte Trübung. Pupillarmembran ist vieI dümner geworden, aber noch ringsherum adhärent. Patient giebt an, besser zu sehen.

21. Juni. Noch besser. Exsudat im Pupillargebiet ganz verschwunden, Pupillarrand aber noeh fixirt, nicht merklich auf Licht reagirend. Kammerwasser ganz klar.

23. Juni. Langsamere Besserung. Noch zarte lineare Hornhauttrübungen, besonders im Centrum. Pupille fängt an wieder etwas beweglicher zu werden. Einzelne hintere Synechien treten etwas hervor, Pupille aber: fast regelmässig rund (kein Atropin). Ophth. Bild nur leicht verschleiert, Papille vielleicht ein wenig geröthet, Gefässe normal. Keine flottirenden Glaskörpertrübungen, noch sonstige Veränderungen im Angengrund.

24. Juni. Injection noch geringer geworden. Hornhaut wie gestern. Pupille (ohne Atropin seit dem 17.) etwas weiter als die linke, ziemlich circulär adhärent, doch bei Lichtwechsel wohl eine leichte Beweglichkeit zu constatiren. Nach einmaliger Atropininstallation wird sie kaum merklich weiter. Angendruck ganz normal.

Pat. wird entlassen mit der Verordnung, zu Hause Natr. salic. weiter zu nehmen.

6. Juli. Injection grösstentheils verschwunden. Pupille immer noch weiter als links (ohne weitere Anwendung von Atropin), leicht unregelmässig, mit Andeutung von hinteren Synechien, auf Lichtwechsel nicht merhlich reagirend. Nach Eserin Verengerung, etwa bis zur Weite der linken, einige Synechien deutlicher hervortretend. Druck normal. Pat. bemerkt ab und zu bewegliche Trübungen; objectiv nur einmal. und sehr undentlich etwas von Glaskörpertrübung wahrgenommen. Augenhintergrund ziemlich klar. Netzhautgefässe noch etwas ausgedehnt.

19. Juli. Pupille immer noch weiter als $L_{\text {., }}$ aber auf Licht reagirend. Feine hintere Synechien. Keine Glaskörperopacitäten nachweisbar. Patient klagt noch über etwas Nebel. R. mit $-3 \mathrm{D} \mathrm{S}^{20 / 70-50 .}$ N. 3 (J.) gelesen.

Soll noch 14 Tage lang 1,0 Natr. salicyl. pro die nehmen. 15. Februar 1882. Patient kommt wieder, weil seit 
ca. 8 Tagen sein linkes Auge an Iritis erkrankt ist, während sich das rechte seitdem sehr gut gehalten bat. Im Uebrigen keine besonderen Beschwerden.

L. leichtes Oedem der Lider; starke Ciliarinjection, starke diffuse Kammerwassertrübnng, Hornhaut in ganzer Ausdehnung etwas matt, besonders in der unteren Halfte, eben merklicher Anfang von Hypopyon, starke grünliche Verfärbung der Iris, Pupille auf Atropin etwas weiter als linls; ibr Rand wegen der Trübung nur undeutlich zu erkennen, nicht sehr unregelmässig. Augendruck gegen $R$. eher etwas erhöht. Finger auf ${ }^{3} / 4^{\prime}$ unsicher gezählt.

R. Nur minimale Reste hinterer Synechien in Gestalt von feinen grauen Pünktchen am Pupillarrand, der übrigens volikommen beweglich ist. Ophthalmosc. normal.

Patient wurde dieselbe Behandlung empfohlen wie fur das andere Auge, welche er zu Hanse unter Leitung seines Hausarztes, Herrn Dr. Ritscher, durchmachte und wobei die Iritis ebenfalls einen günstigen Verlauf nahm. Im folgenden Jahr kam er naeh Göttingen, um Prof. Eichhorst zu consultiren, da er an Zuckerkrankheit'leide, mit den Augen ging es seiner Aussage nach vollkommen gut. Prof. Eichhorst constatirte hochgradigen Diabetes mellitus, spec. Gew. 1,040, etwas Albumin, kein Aceton; in der rechten Lunge beginnende phthisische Verảnderungen. Ord. strenge Diat und Salicylsäure.

Nach gütigem Bericht von Herrn Dr. Ritscher hatte Patient stets einen hohen Zuckergehalt, nie unter $6 \mathrm{pCt}$., der auch durch eine Kur in Karlsbad und alle sonstigen Mittel incl. Natr. salic. aicht wesentlich beeinflusst wurde. Er starb ganz plötzlich an einer profusen Lungenblutung, der Erinnerung nach im Frühjohr 1882, nachdem im letzten halben Jahr einige unbedentende Vorlänfer derselben vorhergegangen waren. Ein Recidiv der Iritis trat nicht auf, auch wurde nichts von Cataract constatirt.

Ob der Diabetes schon zur Zeit, wo Patient hier an Iritis behandelt wurde, bestand, ist leider mit sicherheit nicht mehr zu ermitteln, aber in hohem Grade wahrscheinlich. Der starke Zuckergehalt, die leichte Albuminurie, die Complication mit Phthisis pulmon. deuten alle auf ein langjähriges Bestehen desselben hin. Da Patient keine diabetischen Beschwerden klagte, musste die Krankheit damals leider unentdeckt bleiben. 
Entzündung der Iris und Cornea bei Diabetes mellitus. 221

Fall IV.

Iritis fibrinosa purulenta oc. d., adhaesiva ocs, Diabetes mellitus. Albuminuria levis.

Banquier Fr. aus G. kommt am 7. April 1883 mit der Klage über Abnahme des Sehremögens seit Kurzem, besonders für die Ferne, will früher nicht liurzsichtig gewesen sein.

Bds. Cataracta incipiens, nur leichte Kerntrübung. L. alte Maculae corn. Kein Staph. post.

R. mit $-9 \mathrm{D} \mathrm{S}{ }^{20} / 100$. N.1 (J.) gel. in $3-4 "$. Se fr.

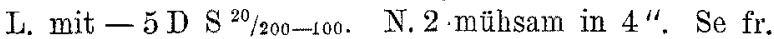

14. Norember 1883. Cataract hat nicht merklich zugenommen. Muss beim Lesen sehr annähern. Leichte Conjunctivitis.

19. Mai 1884. Cataract etwas fortgeschritten. R. mit $-9 \mathrm{D} \mathrm{S}{ }^{20} / 200$ nahez., N. 5 mühsam. L. mit $-5 \mathrm{D}$ Finger in $18^{\prime}$, N. 5 mühsam.

7. Juni 1884. R.Frische Iritis seit 8 Tagen, stärkere Sehstörung seit vorgestern. Geringe Ciliarnenrose. Keine Ursache nachzuweisen. Pat. will keinen Rheumatismus gehabt haben, noch sonst eine Krantheit, ausser Erysipelas eapit. vor einigen Jahren.

R. starke Inject., leichte Chemosis; zarte Fibrinhaut in der Pupille. Augengrund nur wienig zu erleuchten. Känn Finger zählen, aber nicht lesen. L. Auge frei von Entzündung.

Die Combination von Cataracta incipiens mit acquisiter Myopie und der eigenthümlichen Form von Iritis veranlasste nach früleren Erfahrungen die Untersuchung des Urins, insbesondere auf Diabetes. Dieselbe ergab mässige Albuminurie und sehr hohen Zuckergehalt $(10,4 \mathrm{pCt}$.). Keine diabetischen Beschwerden; Appetit gut, aber nicht auffallend stark, kein vermehrtes Durstgefühl. Ein Bruder des Patienten leidet nach dem Bericht seines Arztes ebenfalls an Diabetes mellitus. Ord. Atropin, warme Umschläge, Natr. salicyl. 2 mal tägl. 2;0. Mässige diabetische Diät mit reichlich Fett.

9. Juni. R. Chemosis im Verschwinden. Injection etwas geringer. Kleines Hypop yon. Pupillarmembran. vom innern. Rande etwas abgelöst, der sich etwas mehr dilatirt hat.

11. Juni. R. Pupille eher mehr getrübt, doch scheint sich die Exsudation in der vordern Kammer etwas zusammenzuziehen. Grosse subconj. Blutung. Keine subj. Beschwerden. Atropin ausgesetzt. 
13. Juni. Besser. Exsudation fängt an, mehr vom Pupillarrand zuriekzuweichen. Injection geringer. Zuckerreaction geringer, noch etwas Eiweiss vorhanden.

19. Juni. Injection geringer. Pupille ziemlich frei ron Exsudat, noch etwas erweitert und leicht unregelmässig durch hintere Synechien. Papille noch stark verschleiert.

23. Juni. Auf. Atropin treten viele hintere Synechien hervor, wieder $2 \mathrm{mal}$ tägl. eingeträufelt.

24. Juni. Atropin wird gut vertragen. Pupille erweitert sich.

26. Juni. Zucker eben noch nachweisbar, Spuren von Eiweiss. Dosis des Natr. salieyl. anf 2,0 pro die herabgesetzt, noch einige Tage weiter zu gebrauchen.

15. Juli. Seit 10 Tagen ohne Behandlung. Iritis ziemlich abgelaufen. R. mit $-9 \mathrm{D}$ Finger in 10-11' gezählt, ohne Q1. N.9 (J.) mühsam.

25. September. Iritis völlig abgelaufen. Pupille ohne Atropin leicht oval, frei von Exsudat. Augengrund wenig mehr zu erlevchten wegen diffuser Linsentrübung. Nach Atropinisirung Pupille mässig erweitert, etwas horizontal oval. Feine hintere Synechien fast am ganzen Rand. Linke Pupille etwas besser zu durchleuchten. Sehvermögen der Linsentrübung entsprechend, am rechten Auge jetzt in Gegensatz zu früher wenig Besserung dureh Concavgläser in der Ferne:

R. Finger in $6-7^{2}$, mit $+4 D$ N. 12 mühs.

L. mit $-5 \mathrm{D}$ Finger in $15^{\prime}$ N. 5 sehr mühs. Se beiders. frei.

14. October. Nach Bericht des Hausarztes soll in letzter Zeit wieder ziemlich viel Zucker im Harn gewesen sein. Heute wird er frei von Zucker gefunden.

Am 1. November 1884 erkrankte Patient an schwerer Gangrän des $x$. Fusses, woran er über ein halbes Jahr zu leiden hatte; nach Abstossung einiger Zehen kam der Process zu langsamer Verheilung.

Im Frühjahr 1885, bei schon ziemlich fortgeschrittener Heilung, trat am linken Auge Iritis auf, geringeren Grades als zuvor am rechten, mit mässiger Injection, geringer Kammerwassertrübung und mehreren h. Synechien, aber ohne Hypopyon, die bei warmen Umschlägen und Atropin im Laufe Fon 6-8 Wochen zurückging.

21. Juli 1885. L. Iritis mit Hinterlassung von einigen 1. Synechien vällig abgelaufen. Die Cataract hat indessen 
Entzündung der Iris und Cornea bei Diabetes mellitus. 223

auf beiden Augen Fortschritte gemacht, ist aber noch nicht matur.

31. August. L. ungewöhnlich grosses Hordeolum am oberen Lid, das weiterhin bei einfacher Behandlung bald zur Heilung kam.

12. September. Cataract beiders. noch nicht ganz matur; Pat. zählt R. nur Finger in $3^{\prime}, \mathrm{L}$. in $5-6^{\prime} ;$ R. mit $+4 \mathrm{D}$ N. 17. Worte, L. mit $+4 D$ N. 16 Worte. Kein Rückfall von Iritis aufgetreten.

\section{Fall V.}

Iritis adhaesiva duplex. Opacit. in corp. vitr., Diabetes mellitus.

Pfarrex Joseph 0, $39 \mathrm{Jahr}$, aus H. bei Heiligenstadt. Pat. will vor etwa $1 / 2$ Jahr rasch vorübergehende Augenentzündung gehabt haben, die wenig beachtet wurde und vor 2 Monaten recidivirte. Schon seit etwa 2 Jahren Flimmern und seit 1/2 Jahr bewegliche Trübungen vor dem Auge.

Stat. praes. am 4. Juli 1873. R. einige hintere Synechien und Reste von solehen. Angengrund mässig versehleiert, keine Glaskörpertrïbungen direct nachweisbar, ebenso wenig heerdförmige Veränderungen im Augengrunde. L. keine Reste von Iritis; eine umschriebene Glaskörpertrübung vor der Papille hin und herschwebend. In der Aequatorialgegend nach innen sehr peripherisch einige dunkle Pigmentflecke, vielleicht hämorrhagischen Ursprungs. R. S ${ }^{20} / 70$, L. $\mathrm{S}^{20} / 20$. Ord. Atropin, 3 Blutegel, Sublimatpillen. Patient scheint sich damals nicht weiter vorgestellt zu haben, und kam erst fast 4 Jahre später wieder zur Behandlung wegen erheblicherer Sehstörung, besonders am linken Ange.

6. Februar 1877. R. einige hintere Synechien und feine flottirende Glaskörperopacitäten. I. zahlreiche breite hintere Synechien, feine Pupillarmembran. Glaskörpertrübungen nicht deutlich zn erkennen. Augengrund stark verschleiert. Pupille nach Atropin sehr unregelmässig und nicht maximal erweitert. R. E. S ${ }^{20} / 50-40$ N. 1 (J.) bis 7". Se fr. L. E. $\mathrm{S}^{20} / 200 ;$ mit $+6 \mathrm{D} \mathrm{N} .4$ sehr m. Se fr. Keine Ursache des Leidens zu entdecken. Keine Drüsenschwellungen, noch sonstige Indicien für Lues. 
Ord. Atropin, Jodkalium.

19. Februar. R. S $20 / 30$ nahezu. L. Stat. id., daher L. Iridectomia mit schmalem Messer nach unten. Breites Colobom. Das in der vorderen Kammer zurückgelassene Blut ist schon am Abend bis auf geringe Reste resorbirt. Heilungsverlauf nicht frei von Iritis.

23. Februar. Kammerwasser etwas trübe, am Boden der vorderen Kammer ein graulicher Exsudatstreif. Druckempfindlichkeit.

26. Februar. Auge stark injicirt; vordere Kammer aufgehoben. Leichte streifige Hornhauttrübung in der Ausdehnung des Coloboms und der Pupille. Noch mässige Druckempfindlichkeit.

29. März. Nach mehrmaliger Application des Heureloup und fortwährendem Gebrauch ron Jodkalium und Atropin bessert sich der Zustand allmälig, so dass nur noch geringe Injection zu sehen ist. Neben der Wunde in der Tiefe eine granliche streifige Trübung, sonst Colobom klar. Patient auf Wunsch mit Verband entlassen. Sebvermögen noch sehr unbefriedigend.

20. Juni. Seit der Entlassung traten keine Nachschübe yon Entzündung auf und verlor sich'der Rest ron Injoction allmälig ganz, doch blieb das Sehvermögen des operirten Auges bedeutend herabgesetzt und schlechter als vor der Operation. Zahlreiche hintere Synechion. Colobom ist offen geblieben, aber won einer zarten Membran rollständig ausgefüllt: Neben der Narbe stärkere weisshiche Trübung in den tioferen Hornhautschichten oder auf der Kapsel. Angengrund stark verschleiert. Zählt Finger in ca. 13', mit $+6 \mathrm{D}$ N. 15 mïhsam. R. S ${ }^{20} / 30-20$.

23. Juni 1881. Patient stellt sich erst heute wieder vor, nachdem er inzwischen längere Zeit in anderer Behandlung gewesen war. Nach der letzten Vorstellung hier trat Iritis am rechten Auge auf, an welcher Patient im October bis December 1877 yon Mooren in Düsseldorf behandelt wurde, u: a. mit Inunctionscur. Nachdem die Entzündung ziemlich zurückgegangen war, so dass Patient lesen konnte, trat im Juni des folgenden Jahres (1878) ein neves Recidiv ron Iritis am rechten Auge auf, wegen dessen am 26. October 1878 vonMooren Iridectomie nach oben gemacht wurde. Pat. konnte vor der Operation feinere Schrift nicht lesen Am 5. Tage nach der 
Entzïndung der Iris und Cornea bei Diabetes mellitus. 225

Operation abermals Entzündung, welche $1^{1 / 2} \mathrm{Jahr}$ dauerte und allmälig dazu führte, dass Patient gar nicht mehr lesen konnte. Damals soll, etwa 1 Monat vor der Operation, Diabetes mellitus constatirt worden sein. Seitdem blieb Patient von Entzündung verschont und das Sehvermögen ist an beiden Augen etwa im gleichen Grade gestört.

Stat. pr.: R. Sclerosirende Hornhauttrübung von unten her bis etwas vor die Pupille sich erstreckend; ziemlich schmales Colobom nach 0 , in demselben etwas iritische Auflagerung auf der Kapsel. Linse anscheinend durchsichtig. Keine Injection. Zählt Finger in $16^{\prime}$, mit $+4 \mathrm{D}$ N. 15 (J.) mühsam. L. etwas breiteres Colobom nach unten. Neben der Narbe ein schmaler, quorer Trübungsstreifen, anscheinend in der vorderen Kammer. Colobom übrigens frei. Pupille wenig zu durchleuchten, zarte Pupillarmembran, vielleicht auch beginnende Cataract. Mit $-1 \mathrm{D}$ Finger in $20^{\circ}$, mit $+4 \mathrm{D}$ N. 14. Pat. beobachtet seit der Constatirung des Diabetes strenge diabet. Diät und hat nur wenig diabet. Beschwerden; Durstgefühl jetat nur mässig, schwitat viel, hat keine Abnahme der Kräfte bemerkt.

6. Juli 1881. 24std. Harnmenge $2040 \mathrm{Ccm}$, React. sauer, spec. Gew. 1,024-25, frei von Zucker und Eiweiss, obwohl Pat. ein Brödchen gegessen hat.

Dass der Urin frei von Zucker und Eiweiss, wird auch bei zweimal wiederholter Untersuchung (am 7. Juli u. 9. Juli) bestätigt; am letzten Tage 24std. Menge $1720 \mathrm{Ccm}$. bei 1,027 spec. Gew.

Pat. geniesst seit dem 8. Juli gemischte Kost.

9. Juli. R. Iridectomie nach aussen mit der Lanze. Bei Tageslicht freies, schwarzes Colobom.

17. Juli. Vollkommen normale Heilung. Auge schon kaum mehr injicirt. Mässig breites Colobom nach aussen. Hornhaut davor nicht unerheblich getrubt. Linsenrand sichtbar. Augengrund nicht deutlich zu erkennen, obwohl die Linsenkapsel im Colobom ganz rein ist. Auch - keine Linsentrübung nachweisbar.

19. Juli. Pat. entlassen. Auge fast vollkommen frei von Injection. Zählt Finger in $14^{\prime}$, stenop. in $17 '$; mit $+5 \mathrm{D}$ N. 15 mühsam. Von Diabetes während des ganzen Aufenthaltes Nichts nachweisbar gewesen.

18. Angust. Nach Brief ist Sehvermügen etwas besser.

จ. Graefe's Archiv fiir Ophthalmologie: XXxr. 4. 
Bei gemisehter Kost Zunahme des Körpergewichtes um 8 Pfd. Uebersandter Urin frei von Zucker.

3. October. Pat hat gemischte Kost genossen mit Ausnalume von Zucker und Süssigkeiton und sich dabei wohl befunden. Körpergewicht hat im Ganzen um 14 Pfd, zugenommen. R. Colobom nach aussen sehr schon, aber Hornhaut dayor ein wenig trübe. Papille mässig verschleiert. S könnte nach den opt. Verhältnissen besser sein. R. zählt Pat. Finger in 14', mit +6 D N.14 sehr mülssam. L. Cataract weiter fortgeschritten. Augengrumd nur wenig zu erleuchten. Mit $-1 \mathrm{D}$ Finger in $10^{\prime}$, mit $+6 \mathrm{D} \mathrm{N} .14$ mühsam. Urin hente wieder untersucht. Spec. Gew. 1,014; mit Fehling"scher Lösung, gekocht etwas trubbe, gelbe, lehmartige Ausscheidung, wie os ofter beim Vorhandensoin von Spuren von Zncker rorkommt.

17. Jannar 1882. Uebersandter Urin frei ron Eiweiss und Zncker trotz gemiscluter Kost (mit Ansnalume von Kartofieln und Zucker).

11. October. R. Obj. Befund und Sehvermögen unverändert. L. Cataract fortgeschritten, zählt Finger in $9^{\prime}$, Se frei. Lichtempfinding gat. Urin ziemlich stark zuckerhaltig. (Antidiabet. Diät.)

29. Oetober. Cebersandte Crinprobe znekerfrei.

19. Februar 1884 kommt Patient wieder zur Untersnchung. R. Status idem. L. Cataract als matur zu bezeichnen, zählt noch Finger in $3 \frac{1}{2}$ s. Lichtschein und Projection gut.

Nach brieflichom Bericht yom 7. October 1885 sind licine Nachschübe von Entzündung eingetreten und hat sich das Sehvermögen des $x$. Auges eher etwas gebessert, mit dem linken werden noch Finger in $1^{\prime}$ Entfernung gezählt. Das Allgemeinbefinden ist durchaus befriedigend, kein auffallender Hunger oder Durst, doch etwas reichliches Flüssigkeitsbedürfniss. Durehschnittliche Urinmenge 2 Liter. Der Urin war bei gemischter Kost im Februar 1884 wieder znckerhaltig gefunden worden, weshalb Pat. die strengere Diät wieder aufnahm; doch dauerte es langere Zeit, ehe der Zucker wieder verschwand. Im Jetzten Sommer wurde Wasser von Neuenahr getrunken mit gutem Erfolge, indem jetzt nur hin und wieder Zucker constatirt werden konnte. Pat. selat dic strenge Diät noch weiter fort. 
Entzündung der Iris und Cornea bei Diabetes mellitus. 227

\section{Fall VI.}

Iritis adhaesiva oc, d., Cataracta oc. utr. Diabetes mellit.

Frau Rieke $\mathrm{R}$, 83 Jahr, aus Beverungen.

1. Juli 1884. Beiderseits Cataracta matura dura, vom Aussehen der C. nigra. R. Frisehe Iritis mit hinteren Synechien und Beschlägen der Membrana Descemetii. Patientin in die Klinik aufgenommen, wurde mit Atropin, warmen Umschlägen und Natr. salicyl. int. behandelt, wobei die Iritis in etwa $3 \mathrm{~W}$ ochen mit Hinterlassung einiger hinterer Synechien, aber mit freiem Pupillargebiet zurückging.

Nach Ablauf der Iritis am rechten Ange wird am linken modif. Linearextraction nach oben ausgefübrt (Prof. Deutschmann). Operation durch vollständige Taubheit sebr erschwert. Nach Entbindung des Kerns wird noch etwas Corticalis ausgestrichen, woranf von weiteren Versuchen $\mathrm{Ab}$ stand genommen werden muss, da sich Hyaloidea einzustellen beginnt. Heilungsverlauf protrahirt, andauernde starke Injection, viel lineare Hornhauttrübungen, aber Colobom frei. Ophth. Bild nur durch die Hornhauttrübungen verschleiert. Patientin wird auf fortwälrendes Drängen in diesem $\mathrm{Zu}$ m stande am 10. August 1884 nach Hause entlassen und stellt sich am 21. April 1885 wieder vor. L. Ange seit der Operat. immer roth geblieben, auch jetzt stark injicirt. Multiple hintere Synechien und dünner iritischer Nachstaar durch die ganze Pupille. Augendruck niedrig. R. Iritis abgelaufen, hintere Synechien wie fruher.

24. April. L. Iridectomie nach innen unten mit schmalem Messer; breites Stück Iris excidirt. Von der reichlichen Blutung musste viel zurückgelassen werden.

Heilungsverlanf obne Reaction. Das Blut nimmt allmählig ab, wobei die natürliche Pupille zuletzt ziemlich frei zum Vorschein kommt, während das alte wie das nene Colobom von braunem Exsudat verschlossen bleiben. Pupille ziemlich schwarz, doch nur unrollkommen zu erleuchten, vermuthlich wegen Glaskörpertrübungen. Einzelheiten des Augengrundes nicht zu sehen, zum Theil aveh wegen Enge der Pupille und weil Patientin uusicher fixirt.

Das ungünstige Resultat der Iridectomie veranlasste Prof. Leber am 1. Mai 1885 nochmals auf die Anwesenheit ron Diabetes zu untersuchen, obwohl die 83 jährige Patientin 
sich für im Uebrigen völlig gesund hielt. Der Urin soll früher sehon eímal mit negativem Resultat untersucht sein, worubber aber keine Notiz im klinischen Journal zu finden ist. Jetzt ergiebt die Untersuehung starken Zuckergehalt. Patientin erbält, 2 Mal täglich 2,0 Natr. salieyl., doch ohne strenge Diät, zu welcher sie kanm zu bewegen gewesen wäre. Sie klagt nicht iuber ihr Befinden; ihr Appetit ist ziemlich stark, sie isst gern Sussigkeiten, hat keinon auffallenden Durst, doch giebt sie auf Befragen za, dass sie zeitweise viel Wasser trinken müsse.

Bei der Entlassung, am 21. Mai 1885, enthält der Urin noch 3,5 pCt. Zncker. L. zählt Patientin mit $+10 \mathrm{D}$ Finger in $3-4^{\prime}$, das Gesichtsfeld scheint erheblich beschränkt, genaue Prïfung nicht müglich. R. Sehvermögen durch die Cataract etwa im gleichen Grade gestort wie L.

\section{F'all VII.}

Residua Iritidis, Cataracta duplex, Atroph. n. opt., Ablatio retinae et chorioideae, Diabetes mellitus.

Heinrich Ludolf H., 65 Jahr, Cantor in Einbeck, stellt sich vor wegen Abnahme des Sehvermögens, besonders am linken Auge.

12. Juni 1878. R. Cataracta ineip, schmalstreifige Trübungen in der Corticalis, Kern noeh durchsichtig; Augengrund ziemlich verschleiert; Papille ron schmalem, hellen Ring umgeben. Geringe Reste ron Iritis in Gestalt von eimigen Pigmentpünktchen auf der Kapsel.

I. Ausgedehnte h. Synechien, nur der äussere obere Theil des Pupillenrandes frei. Cat, incip. Geringe Ciliarinjection. R. mit $-5,5 \mathrm{D} \mathrm{S} 1 \% 100-70$, mit $+4 \mathrm{D}$ N. 9 (J.) mühs. L. Finger in $4-5^{\prime}$ gezählt, mit Gläsern nicht besser, mit $+4 \mathbf{n}$ N. 18 (J.) Buchst. Se scheint beiders. frei.

Ueber die Entstehung der Iritis weiss Patient wenig Angaben zu machen; er erinnert sich, vor einiger Zeit vorübergehend an Augenentzündung gelitten zo haben, über die etwaige Ursache derselben ergeben sich aber keinerlei Anhaltspunkte. Patient, ein grosser starker Mann, will sonst immer gesund gewesen sein und hat keine anderweitigen Klagen.

Nach einem Jahr, wo sich Pat. am 9. Juni 1879 wieder vorstellt, ist die Cataract $R$. erheblich fortgeschritten, L. nahezu matur. Selhrermögen R. mit $-5,5 \mathrm{D}$ Finger in 8 , 
Entzündung der Iris und Cornea bei Diabetes mellitus. 229

N. 15 (J.) sehr mühs., Se frei. L. mit - 5,5 D Finger in $1 / 2^{\prime}$, Se frei, Lichtschein und Project, gut. In Anbetracht der beträchtlichen hinteren Synechien wird als Vorbereitung zur Extraction am 9. Juni Iridectomie nach unten gemacht. Sehr starke Blutung aus der Conjunctivalwunde, vordere Kammer ganz voll Blut, wovon das meiste zurückgelassen werden muss. Druck auffallend niedrig.

Heilungsverlaut ganz befriedigend. Patient wird, nachdem die reichliche Blutung in die vordere Kammer völlig resorbirt ist, mit freiem Colobom and ganz geringer Injection nach 12 Tagen entlassen; zählt mit 5,5 D Finger in $2^{1 / 2}-3$.

Am 20. October erscheint die Cataract matur, S auf Wahrnehmung von Handbewegungen reducirt. Lichtsinn ist aber jetzt - auffallender Weise - nicht mehr ganz normal; bei heller Lampe Handbewegungen nur geradeaus wahrgenommen; Projection für mittlere Lampe nach innen unten sehr unsicher.

L. modif. Linearextraction nach oben, wobei die $a b-$ geflachte und verkleinerte Linse in geschlossener Kapsel durch einfaches Sturzmanöver' entbunden wird. Erst beim Reinigen der Wunde von abgestreiftem Pigment kommt es in Folge starken Pressens zu einem geringen Glaskörperverlust, der aber nachher sofort wieder steht. Wundränder schliessen gut, sehr grosses Colobom.

Das Auge bleibt lange roth und gereizt, in Folge ron leichter Iriseinklemmung am inneren Wundwinkel.

Am 10. November noch etwas Injection; breites Colobom nach unten und nach oben, Pupillargebiet frei, Glaskörper leicht getrübt. Die ophthalmosc. Unters. ergiebt jetzt eine seichte Excavation der Papille vom Aussehen der Druckexcavation, mit starker Verfärbung und mässig breitem hellem Ring um dieselbe. Angendruck beiderseits ganz normal, war auch früher nie erhöht gefunden worden, auch niemals sonstige glaucomatöse Erscheinungen. Die Natur der Excavation bleibt also vorläufig dunkel. Pat. zählt mit $+5 \mathrm{D}$ nur Finger in 4-5.

Am 30. December. Sehvermögen unverändert. Am inneren Wundwinkel etwas stärkere Vortreibung der eingeheilten Irispartie. R. Pupille auf Atropin maximal weit, von Resten der früheren Iritis nicht das Geringste zu erkennen. 
11. Januar 1880. R. Modif. Linearextraction mit Chloroform, schlechte Narcose. Linse geschrumptt, Kapsel mit scharfem Häkchen eingerissen, Linse durch Sturzmanöver entbunden. Kein Glaskörperworfall.

21. Januar. Auge von Anfang an nicht reizlos; Pupillarrand allmälig leicht adhaerent. Kammerwasser nicht ganz klar, hente leichtes Hypopyon, etwas Chemosis, Wunde zart belegt und von ihr ein Paar gelbliche Fetzchen in die vordere Kammer hineinragend. Deshalb in Narcose die Wunde mit Anel'scher Sonde eröffnet, das Kammerwasser abgelassen und die Wundränder mit $2 \mathrm{pCt}$. Carbolsäure betupft.

11. Februar. Nach mehrmaliger Eroffnung und Reinigung der Wunde erhebliche Besserung und Rückgang der Entzïndung. Auge jetzt ziemlich abgeblasst. Dünne Pupillarmembran, die zwar in der Witte zu durchleuchten ist, aber rom Augengrund nichts erkennen lässt.

7. Mai, R. Entzündung abgelaufen. Vollständiger Pupillarverschluss, Kammer enorm seicht, Iris bucklig vorgetrieben; mit $+6 \mathrm{D}$ Finger in $8^{\prime}$ gezählt. Iridectomie nach unten, wobei ein kleines und wie es scheint, zum grössten Theil freies, schwarzes Colobom entsteht.

8. Mai. R. Colobom unrein. Iritis.

Auf Calomel in refr. dos. weicht die Iritis ziemlich rasch, doch bleibt das Colobom bis anf eine minimale freie Litoke tribe und es wird keine Besserung des Sehvermögens erzielt. Patient wird am 23. Mai nach Hause entlasson.

10. November 1880. R. Ange noch immer etwas injicirt. Das neue Colobom ist vollig zugegangen. Das obere stark verengt und durch eine nicht sehr dicke Membran verschlossen. Augendruck normal. Auge soll ofters noch mehr geröthet sein als heute; weitere Operation daher noch verschoben.

Als sich der Kranke am 19. Jannar 1881 wieder vorstellte, war die Injection an beiden Augen bis auf geringe Reste zurückgegangen. Am rechten Auge war der Befund wie er nach Extraction mit nachfolgender Iritis sich häufig darbietet; am linken Auge dagegen war, dem Kranken unbemerkt, durch Hinzutritt ausgedehnter Netzhaut- und Aderhautablösung, bei freigebliebener Pupille der Rest von Sehvermögen völlig verloren gegangen. $R$. noch immer die grösseren Gefässe ein wenig ansgedehnt und geringe, mehr oberflächliche Cireumcornealinjection. Cornea in unteren Abschnitt etrvas matt. 
Entzündung der Iris und Cornea bei Diabetis mellitus. 231

Beide Colobome vollständig rerschlossen. Iris stark vorgebuckelt. Pupille nicht zu erleuchten. Augendruck nicht merklich erhöht. Mit $+6 \mathrm{D}$ Finger in $1^{1 / 2} 2^{\prime}$ gezählt. Lichtschein und Projection gut. L. ebenfalls etwas Circumcornealinjection. Pupille weit und frei wie früher. Eingeheilte Iris nicht sehr vorgetrieben. Augendruck nicht merklich gesteigert. Medien ziemlich klar. Im Glaskörper nur sehr zarte Trübungen and Streifen, aber nicht flottirend. Nasalwärts grosse Netzhautablösung mit deutlich sichtbaren Gefässen in Gestalt einer prall gespannten Blase. Temporalwärts ein ebenso stark hervorspringender Buckel, an dem man aber keine Gefässe bemerkt. Die Papille ist zwischen beiden Buckeln ganz deutlich zu sehen mit der schon früher erwähnten Excavatioṇ. Hit focaler Beleuchtung sind die beiden Buckel ebenfalls deutlich zu sehen; an ihrer braunen Farbe lässt sich erkennen, dass es sich nichtnur um Abiösung der Netzhaut, sondern auch der Chorioidea, resp. des Ciliarkörpers handelt. Nasalwärts schimmert die Innenfläche der letzteren durch die Netzhaut hindurch und sieht man deren Gefässe ganz dicht über das braune Pigmentepithel hinüberziehen; temporalwärts liegt die Aderhant frei, obne von Netzhautgefässen bedeckt za sein (was wohl auf eine ansgedehnte periphere Zerreissung der Retina hinweist). In geringem Abstand vor ihr bemerkt man noch ein zartes weissliches Hüutchen ohne Gefässe. Sehvermögen bis auf schwachen Lichtschein aufgehoben.

Durch Durchtrennung der Membran mit Wecker'scher Scheere in verticaler Richtung wird $R$. wesentliche Verbesserung des Sehvermögens (Fingerzählen in $5 \%$ erzielt. Heilung der Wunde erfolgt absolut reizlos.

3. Februar Erst gestern Naclimittag, eben vor der Entlassung wird in Erfabrung gebracht, dass der Patient während seines Hierseins einen enormen Appetit entwickelt und sich heimlich grosse Quantitäten Mettwurst hatte zutragen lassen. Auch zu Hause soll er schon viel Wasser getrunken haben und ofters des Nachts aufgestanden sein, um zu uriniren. Doch hatte ihn dies nicht weiter beunruhigt und er hatte in keiner Weise über diabetisclie Symptome geklagt. Er ist corpulent und wohl genährt, giebt auch anf Befragen keino Abnahme der Kräfte zu. Harn zeigt 8,25 pCt. Zucker, kein Eiweiss.

25. November. R. nur noch sehr wenig Injection. Beide 
Colobome nach oben und unten vollkommen verschlossen. Etwas Hormhauttrübung. Augendruck normal. Sieht nur Bewegungen der Hand. Lichtschein nur für niedere, nicht niederste Lampe. Projection fehlt nach oben. L. kein Lichtschein. Patient fühlt sich kräftig und will weder auffallenden Durst noch Hunger haben, muss aber 2-3mal des Nachts anfstehen. Urin bei spec. G. 1,042 enthält 8,8 pCt. Zucker.

Zu einer Behandlung des Diabetes in seiner Heimath wollte sich Pat. nicht verstehen.

Im nächsten Jahre kam er wieder und wurde trotz der schlechten Prognose zu einem letzten Curversuch in die Klinik aufgenommen.

18. März 1882. R. Bewegungen der Hand. Lichtschein und Projection erst nach längerem Aufenthalt im dunklen Zimmer gut, vorher nicht genügend. L. absolute Amaurose. Urin bei spec. G. 1,043 enthält 6,7 pCt. Zucker und Spuren von Eiweiss.

25. März. Nachdem 2 Tage lang mässige diabetische Diät beobachtet war und 2 mal tgl. 2,0 Natr. salicyl. genommen, wurde nur noch 0,5 pCt. Zucker gefunden, bei spec. G. 1,013.

26. März. R. einfache Iridotomie in horizontaler Richtung.

7. April 1882. Am Tage nach der Operation zeigte sich dio gemachte Oeffnung vollständig von Blut verlegt, das sich allmälig resorbirte, aber olne dass eine freie Lüeke zum Vorschein kommt. Hinter dem Spalt treten jetzt Schwarten hervor, nur in der Mitte noch ein wenig Blut. Pat, erhielt während seines hiesigen Aufenthalts mässige diabetische Diät. Dosis des Natr. salicyl. wird wegen Ohrensausen von 4,0 anf 2,0 reducint. Harn zeigt bei spee. G. von 1,030 wieder beträcht. lichen Zuckergehalt. Subjectives Wohlbefinden, (Pat. hat sich wahrscheinlich nebenher Amylaceen zu verschaffen gewusst.)

22. April 1883. Pat. stellt sich noch einmal vor. R, wieder völliger Pupillarverschluss. Lichtempfindung nur für helle Lampe. Augendruck normal. 'L. Auge weich. Hornhaut diffus parenchymatös getrübt. Oberfläche ziemlich gut spiegelnd. Das grosse Colobom noch eben zu erkennen. Kein Lichtschein. Urin spec. G. 1,032, enthält viel Zucker, kein Eiweiss.

21. September 1884. Nach Zeitungsanzeige ist Pat. heute gestorben. 
Entzündung der lris und Cornea bei Diabetes mellitus. 233

\section{Fall VIII.}

Resid. iritidis, Cataracta glaucomatosa in oc. dextr., Cataracta senilis, postea Atroph. n. opt. in oc. sin., Diabetes mellitus.

Baron Detlef v. U., $67 \mathrm{Jahr}$, aus Berlin, berichtet, dass er schon vor Jahren von Prof. v. Graefe in Berlin an Iritis beider Augen behandelt worden sei und dass er eine für das $r$. Auge vorgeschlagene Iridectomie nieht habe ausführeu lassen, worauf es allmälig vollkommen erblindete. Dasselbe war später ziemlich frei von Entzündung, wäbrend Patient kürzlich wegen Rückfall von Iritis am linken Auge in Behandlung war.

16. Juli 1878. R. Status glancomatosus, Ausgänge von Iritis, verkalkte Cataract, keine vordere Kammer. Absolute Amaurose.

L. Ausgänge von Iritis. Dünne Pupillarmembran, mit nur zwei kleinen freien. Stellen. Finger in $10^{\prime}$, mit Concaygläsern nur wenig besser. In der Nähe N.5 (J.) mühsam. Se frei.

Da Patient wenig geneigt ist, die ihm schon von anderer Seite vorgeschlagene Iridectomie machen zu lassen, wird er zunächst in Beobachtung behalten und eine Jodkaliumlösung als Tropfwasser verordnet. Bis Ende des Monats hatte sich das Sehvermögen eher etwas gehoben, dagegen trat am 7. August 1878 ein starker Rückfall der Iritis ein, welcher das Sehen bis auf quantitative Lichtempfindung aufhob. Allgemeinbefinden ungestört, ätiologisch nichts $\mathrm{zu}$ ermitteln.

Ord. Natr. salicyl. $3 \mathrm{Mal}$ tgl. 2,0, Ungt. ciner. an die Stirn, warme Umschläge und Heurteloups.

Die Entzündung besserte sich rasch und war nach drei Wochen völlig abgelaufen, doch blieb das Sehvermögen gegen früher nicht unerheblich herabgesetzt.

23. August. L. Finger in 9-10. No. 12.

Am Abend des 4. September wieder starker Reizzustand mit Schmerzen, der sich bis zum nächsten Tage nach warmen Umschlägen bedeutend bessert; doch bleibt das Auge wieder einige Zeit leicht entzündet und es tritt eine weitere Verschlechterung des Sehvermögens ein.

23. September. L. Finger in 5-6, Buchstabirt No. 17. Bewegingen der Hand nach allen Seiten wahrgenommen, Finger nach unten nur unsicher oder gar nicht gezählt. Keine Ciliarinjection. Iris ziemlich stark vascularisirt. Vordere Kammer sehr seicht. Vollständige Pupillarmembran 
mit nur zwei kleinen weniger dichten Stellen am Pupillarrand. Augendruck etwas hoch.

L. Iridectomie mit schmalem Nesser nach unten innen erzielt ein mässig grosses dreieckiges Colobom. Sphinkter stehen geblieben.

9. October. Heilung normal erfolgt, die geringe Blutung wurde rasch resorbirt und das rein aussehende Colobom hat sich noch etwas erweitert, so dass die Papille nur wenig verschleiert sichtbar ist. Keine Veränderungen im Augengrunde zu bemerken. Sehvermögen den optischen Verhältnissen nicht ganz entsprechend.

L. Finger in $15^{\prime} ;$ mit +6 No. 9 mühsam, Worte ron No. 6 und 5 , ohne Convexglas aber fast ebenso gut.

Bei einer späteren Prüfung am 23. October fand sich dem entsprechend, dass mit Concavgläsern anf die Ferne besser gesehen wurde: mit $-3 \mathrm{D}$ Finger in ea. $16^{\prime}$ gezählt; in der Nähe No. 10 (J.) mühsam (ohne Glas).

Patient stellt sich erst am 20. Juli. des folgenden Jahres (1879) wieder vor und berichtet, dass er unterdessen wieder eine Hntzündung gehabt labe, von der jetzt nichts mehr zu bemerken ist. Das Colobom ist frei geblieben, nur vielleicht etwas enger, aber mehr Linsentrübung. Papille nur stark versehleiert zu sehen. L. Finger in ca. 9! Worte ron No. 16.

Bis zum 28. September 1881, wo der Kranke sich wioder zur Untersuchung einfindet, stellten sich noch mehriach Nachschübe der Entzündung ein, doch ohne nennenswerthe Residuen in der Pupille zu hinterlassen. Sehvermögen abermals gesunkèn:

L. Finger in $5-6^{\prime}$, mit Gläsern nicht besser. Ohne Glas Buchstaben von No. 19. Colobom ziemlich frei geblieben, nur ganz zarte Auflagerung auf der Kapsel, aber die Linse entschieden mehr getrübt.

In den beiden folgenden Jahren trat kein Rückfall der Entzündung ein und nahm das Sehvermögen nur sehr wenig $a b$, so dass dasselbe am 16. Juli 1882 ganz unverändert gefunden wurde und am 10. Juni 1883 L. noch Finger in 6-7' gezählt, aber nichts von No. 19 mehr gelesen wurde.

Am 10. Juli 1884, wo das Sehvermögen weiter abgenommen hat, ist notirt: Cataracta fere matura. Noch ziemlich viel ungetrubte Corticalis. Lichtschein und Projection gut.

11. Juli 1884. Modiffinto Linearextraction nach oben 
Entzündung der Iris and Cornea bei Diabetes mellitus. 235

(Prof. Deutschmann). Eine gleichmässige dünne graue Schicht bleibt im Colobom zurüek, die nicht heransgebracht werden kann.

6. Ảugust. Heilungsverlauf normal, aber Resultat zunächst sehr unrein durch Trübung in äer Pupille. Am Tage nach der Operation zeigten sich sowohl das neu angelegte Colobom nach oben wie das alte nach unten von einer ganz gleichmässigen gelblichen Schicht ausgefüllt, die weniger zurückgelassenen Corticalresten als iritischen Membranen mit etwas verdickter Linsenkapsel zu entsprechen scheint. Dieselbe bat sich auch weiterhin nicht merklich resorbirt, lässt sich mit dem Spiegel durchleuchten, doch sind keine Einzelheiten des Augengrundes zu erkennen. Mit $+8 \mathrm{D}$ Finger in $2^{\prime}$ gezählt. Pat. wird vorläufig entlassen und in 3-4 Wochen zur Discision wieder bestellt.

1. September. Durchschneidung der Pupillarmembran mit der Wecker'schen Scheere. Durch 2 vertical auf einander stehende Schnitte wird ein breiter klaffender Spalt erreicht. Geringer Bluterguss in die vordere Kammer.

16. September. Langsame Resorption des Blutes, wobei die entstandene Oeffnung durch ein dünnes Fibrinbäutchen wieder verschlossen wurde.

Patient vermag auch mit Staargläsern nur Personen und sonstige grössere Gegenstände in ihren Umrissen wahrzunehmen, aber nicht deutlich Finger zu zählen. Augengrund roth zu erleuchten. Das Sehvermögen scheint den optischen Verhältnissen nicht zu entsprechen, so dass eine Complication mit einer tiefer liegenden Veränderung vermuthet wird. Trotzdem wird der Nachstaar operativ beseitigt, indem, nach vergeblichem Versuch mit einfacher Incision mit dem Sichel'schen Messerchen, eine nochmalige Durchschneidung der Pupillarmembran mit der Wecker'schen Scheere vorgenommen wird, durch welche jetzt ein breiter bleibender Spalt entsteht.

Ophth. unmittelbar nachher vollkommen rothes Licht zu erhalten, aber der Glaskörper erscheint focal beleuchtet hinter der schwarzen Pupille nicht vollständig klar.

Nach beendigter Heilung ist am 25. September 1884 dio Papille ziemlich deutlich zu sehen und exscheint auffallend stark weiss yerfärbt. Ob Excavation vorhanden, ist nicht sicher zu entscheiden, jedenfalls keine tiefe. 
L. mit $+10 \mathrm{D}$ Finger in $1-2^{\prime}$ mühsam gezählt. Se nach allen Seiton stark eingeengt, nur nicht nach oben.

Am 1. Oetober wurde Pat. entlassen. Im folgenden Jahr kam er am 6. August 1885 wieder nach Gottingen. Die natürliche Pupille erscheint jetzt völlig rein, das untere Colobom etwas zu durchleuchten. Ophth. Bild fast ganz klar. Mässig breiter Entfărbungssaum um die Papille nach innen. Papille rein weiss, ganz seicht excavirt, Gefässe nicht geknickt, Arterien ein wenig eng, Excavation sicher nicht glaucomatös; Augendrnck normal.

Die auffällige Coincidenz der doppelseitigen Iritis and Cataract mit Sehnervenatrophie veranlasste nach früheren Erfahrungen bei dem Falle VII auf Diabetes zu untersuchen, der sich in der That herausstellte. Urin blassgelb, giebt bei der Trommer'schen Probe sebr starke Zuckerreaction, ist frei von Eiweiss. Pat. giebt an, viel Wasser zu trinken, muss Nachts ofters aufstehen, isst viel Süssigkeiten, befindet sich aber sonst rollkommen wohl. Der vermehrte Durst besteht angeblich seit 2 Jahren, aber wahrscheinlich schon viel länger. Da der Kranke nicht tuber diabetische Symptome geklagt hatte und die vorhergegangene Iritis und das vorgerückte Lebensalter die Entstehung der Cataract genügend erklärten, war bis dahin die Untersuchung des Urins unterblioben.

\section{Fall IX.}

Haemorrhagiae in Corp. vitr. dextr., Retinitis haemorrhagica sin., Iritis serosa et Status glaucomatosus in oc. utr., Diabetes mellitus, Albuminuria, Cirrhosis hepatis, Pericarditis.

Amtsgerichtsrath M., 53 Jahr, aus Braakel bei Höxter, soll nach dem Bericht seines Arztes seit einer Reihe von Jahren an Leberhyperaemic gelitten haben. Vor einem Jahr stellte sich Bronchialkatarrh mit Blutauswurf ein und wurde Diabetes mellitus constatirt. Seit Oktober 1882 war kein Zucker im Urin mehr nachweisbar, wohl aber ab und an Eiweiss. Vom November 1882 bis Febrnar 1883 traten wiederholt asthmatische Anfälle, von Bronchialkatarrb begleitet auf, neben endo- und pericarditischen Erseheinungen nnd Anasarea der unteren Extremitäton.

Etwa 14 Tage vor Ostern vorübergehende Sehstörung am 
Entzündung der Iris und Cornea bei Diabetes mellitus. 237

1. Auge von etwa $1 / 2$ stündiger Dauer. 14 Tage später plötzlich starke Verdunklung an demselben Auge, wobei aber grössere Gegenstände noch unterschieden wurden. Später spontan einige Besserung. Vor 14 Tagen trat schmerzhafte Entzïndung des Auges auf, seitdem $\mathrm{S}$ ziemlich vollständig aufgehoben. R. keine Störung bemerkt.

14. Juni 1883. St. pr. R. Ophth. nur einige wenige ganz kleine, rundliche Extravasate in der Netzhautperipherie. E. S ${ }^{20} / 40$; mit $+2,5$ D No. 1 etwas mühs. Se fr.

L. Mässige Ciliarinjection, Kammerwassertrübung, Pupille auf Atropin ziemlich weit, Angendruck etwas erhöht, Augengrund nicht zu erleuchten. Bewegungen der Hand bei Lampenlicht nur nach innen, Lichtschein niederste Lampe. Urin hellgelb, enthält ziemlich viel Eiweiss. Keine Zuckerreaction, nur etwas starke, trübe Reduction. Keine Eisenchloridreaction.

Patient wurde zur Anfnahme in die Klinik bestellt, wozn. er sich am 25. Juni 1883 einfand. Inzwischen hatte sich am R. Auge eine ausgesprochene Retinitis haemorrhagica entwickelt mit starker Hyperaemie, streifigen Extravasaten und einigen weissen Flecken in der Retina. Sehvermögen stärker herabgesetzt. R. $\mathrm{S}^{20 / 200-100}$, mit $+2,5 \mathrm{D} \mathrm{N} .7(\mathrm{~J}$.$) .$

L. Entzündung geringer; Augendruck noch etwas gesteigert; Bewegungen der Hand.

Urin blassgelb. Spec. G. 1,013. Spuren von Eiweiss und von Zucker.

26. Juni. Consultation mit Prof. Ebstein, der folgenden Befund notiren liess: „Vergrösserung des Herzens, besonders des 1. Ventrikels. Cirrhosis hepatis hypertrophica, ziemlich starke Vergrösserung der Leber mit etwas höekeriger Oberfläche; leichte, unbestimmte Herzgeränsche, wahrscheinlich pericarditisehen Ursprungs. Pat. früher viel stärker gewesen. Nichts von Gicht. Vor Jahren Sehanker, auch einmal Tripper. Jetzt leichte Vergrösserung der Cubital- und Cervicaldrüsen. Kleine Narbe im Pharynx. Kein dentlicher Milztumor." Augen unverändert. Ord. KJ und mässige diabet. Diät mit Fett.

26. Juni Abends. Seit heute Morgen beträchtliche Verschlechterung des Sehrermögens am rechten Auge, worüber Pat. natürlich sehr aufgeregt ist. Ophth. wenig Aenderung; die venöse Hyperämie R. etwas stärker, einige frische weisse 
Plaques. Einer der weissen Flocke sitzt nach a. o. ron der Papille, ein Gefäss deckend, die andern nach unten. In der Gegend der Macula nichts Auffallendes; in der Peripherie einige zerstrente rundliche Netzhautblutungen. $R$. Finger in $6^{\prime}$ gezahlt, mit +3D N.19 (J.), Se frei. L. Bewegungen der Hand. Ord. trockener Sehröpflkopf.

27. $J u_{j}$. R. Stat. id. L. Injection eher etwas geringer.

Pat. klagt über Schlaflosigkeit, wie überhaupt in der letzten Zeit. Urin enthält sehr spärliche kurze cylindrische Gebilde mit myelinartigen Tröpfchen und wenige, feinkörnige Elemente, vielleicht degenerirte Epithelzellen. Ord. KJ weiter, links Eserin und warme Umschlage, Fussbader.

28. Juni. $R$. noch stärkere Ausdehnung der Netzhautvenen. Finger nur in $5-6$ '.

30. Juni. R. Gestern und heute Stat. id. Schlaf besser.

1. Juli. R. S Stat. id. Ophth. weniger Blatungen; die drei weissen Plaques wie früher, dazu unterhalb der Papille viele kleine weissliche Fleckchen. L. Kanm Bewegungen der Hand. Lichtschein mittlere Lampe. Noch immer leichte Iritis und Angendruck etwas hoch.

3. Juli. R. Stat. id. L. Injection etwas geringer. Augendruck weniger hoeh. Urin ron 1,023 spec. G,, zeigt bei der Zuekerprobe etwas trübe Ausscheidung; bei Eiweissprobe stärkere Trübung als früher.

5. Juli. R. Stat. id., nur ophth. mehr weisse Flecke. Ord. ausser KJ noch Acid. carbol. (0,25 pro die) innerlich.

9. Juli. R. Ophth. die weissen Flecke werden deutlicher und grösser, die Blutungen kleiner, das ganze Bild wird klarer. L. noch immer mässig starke Injection. Wegen Schlaflosigkeit 1,0 Chloral, worauf ziemlich gut geschlafen wird.

11. Juli. Kein Chloral, sehr schlechte Nacht. Gesicht etwas gedunsen. Puls sehr frequent. Augen unverändert, nur das 1. Ange wieder härter. Urin von 1,019 sp. G., klar, weingelb. Eiweiss: masssige Trubung. Kein Zucker.

12. Juli. Nach Chloral besser geschlafen. Consultation mit Prof. Ebstein: Pericarditischer Erguss, Herzdämpfung mehr verbreitert, leichte Geräusche über dem Herzen. Leber wie früher.

Ophth. $\mathrm{R}$. ausgesprochenes Bild der Ret. albumin. Blutungen laben in letaten Tagen wieder zugenommen, die weissen Flecke aber yorherrschend. L. unverändert. 
Entzündung der Iris und Cornea bei Diabetes mellitus. 239

Pat. wird zur Kur nach Driburg geschickt.

26. September. Pat. kommt hente wieder, nachdem er 8 Wochen mit gutem Erfolg in Driburg gewesen, da seit circa 8 Tagen auch am r. Auge Entzündung und Druckgefühl aufgetreten ist, nachdem das Sehvermögen sich vorher etwas gebessert hatte, so dass Pat. allein spazieren gehen konnte. Seit dem Auftreten der Entzündung hat sich aber $\mathrm{S}$ wieder sehr versehlechtert.

R. ausgesprochener Status glancomatosus. Auge hart, mässige Ciliarinjection. Pupille etwas weit, aber noch reagirend. Auge nicht zu erleuchten. Bewegungen der Hand. Lichtschein nahezu niederste Lampe. L. Ange sehr hart, Iris stark vascularisirt. Pupille unregelmässig. Kein Lichtschein. Urin enthält wenig Eiweiss und Spuren ron Zucker.

27. September. R. Scler otomie nach oben.

7. Oetober. Heilungsverlauf ganz befriedigend. Auge nur noch wenig injicirt, eher weniger, als vor der Operation. Pupille von mittlerer Weite, ein wenig unregelmässig, auf Licht prompt reagirend. Am Boden der vorderen Kammer noch ein circa $1 \mathrm{Mm}$ hohes Hyphäma. Augendruck bedeutend heruntergegangen, jetzt nahezu, aber noch nicht ganz normal. Pupille zu erleuchten. Bewegungen der Hand im ganzen Gesichtsfeld; noch keine Finger gezählt. L. unverändert.

8. October. Herzdümpfung nach $r$. hin nicht mehr verbreitert, geht nach 1. 1-2 Finger über die Mamillarlinie. Keine Geräusche mehr. Leber mässig vergrössert, leicht höckerig. Puls an der Radialis gespannt. Entlassen.

Nach ärztlichem Bericht rom 22. October 1885 blieb das Sehvermögen anch weiterhin an beiden Augen verloren, während die in Driburg erlangte Besserung des sonstigen Befindens sich erhielt. Das Körpergewicht hatte bedeutend zugenommen und Zucker and Eiweiss waren verschwunden. Ein Brief des inzwischen nach einem anderen orte übergesiedelten Patienten seilbst vom 23. October d. J. bestätigt den guten Zustand seines Allgemeinbefindens, Körpergewicht 175 Pfund, asthmatische Beschwerden in der letzten Zeit nicht wiedergekehrt. Dagegen sei jede Lichtempfindung erloschen, die Augen übrigens frei ron Schmerzen, nur etwas druckempfindlich.

Aus dem oben mitgetheilten reichhaltigen Material scheint mir kein anderer Schluss gezogen werden zu 
konnen, als dass die Iritis unter diejenigen Erkrankungen des Auges gerechnet werden muss, welche in einem $A b-$ hängigkeitsverhältniss zu dem Diabetes mellitus stehen. Die Häufigkeit ihres Zusammentreffens ist $z u$ gross, als dass sie auf blossen Zufall geschoben werden konnte. Ueber die besondere Art dieses Abhängigkeitsverhåltnisses lässt sich zur Zeit noch keine sicher bewiesene Angabe machen und sind darüber weitere Untersuchnngen $a b-$ zuwarten. 